\title{
Regularized Extremal Shift in Problems of Stable Control
}

\author{
Vyacheslav Maksimov* \\ Ural Federal University and \\ Institute of Mathematics and Mechanics, \\ Ural Branch of the Russian Academy of Sciences, \\ Ekaterinburg, 620990 Russia \\ maksimov@imm.uran.ru
}

\begin{abstract}
We discuss a technical approach, based on the method of regularized extremal shift (RES), intended to help solve problems of stable control of uncertain dynamical systems. Our goal is to demonstrate the essence and abilities of the RES technique; for this purpose we construct feedback controller for approximate tracking a prescribed trajectory of an inaccurately observed system described by a parabolic equation. The controller is "resource-saving" in a sense that control resource spent for approximate tracking do not exceed those needed for tracking in an "ideal" situation where the current values of the input disturbance are fully observable.
\end{abstract}

Keywords: parabolic equation, stable control, extremal shift.

\section{Introduction}

In the present work, the problem of tracking a solution of a system with distributed parameters is discussed. The essence of this problem can be formulated in the following way. A parabolic equation is considered on a given time interval $T=\left[t_{0}, \vartheta\right], \vartheta<+\infty$. The solution of this equation $w(\cdot)=w(\cdot ; v(\cdot))$ depends on a time-varying control $v=v(\cdot)$. This solution is inaccurately measured at frequent enough time moments. It is required to organize a control process for the equation by the feedback principle in such a way that it is possible to preserve given properties of the solution. The quality of the solution constructed is estimated by the distance from a given (prescribed, standard) solution $x(\cdot)$. The latter is a solution of the parabolic equation generated by some input $u=u(\cdot)$. The problem in question is treated as the problem of constructing a control $v=v(\cdot)$ providing the retention of the trajectory $w(\cdot)=w(\cdot ; v(\cdot))$ nearby $x(\cdot)$. This is the conceptual statement of the control problem under consideration.

* This work was supported in part by the Russian Foundation for Basic Research (No. 11-01-12112-ofi-m), by the Program on Basic Research of the Presidium of the Russian Acad. Sci. (No. 12-P-1-1012) and by the Program for support of leading scientific schools of Russia (No. 6512.2012.1).

D. Hömberg and F. Tröltzsch (Eds.): CSMO 2011, IFIP AICT 391, pp. 112-121, 2013.

(C) IFIP International Federation for Information Processing 2013 


\section{Problem Statement}

Let $H$ and $V$ be real Hilbert spaces. The space $V$ is a dense subspace of $H$ and $V \subset H \subset V^{*}$ algebraically and topologically, $(\cdot, \cdot)$ stands for the inner product in $H,\langle\cdot, \cdot\rangle$ stands for the duality relation between $V$ and $V^{*}$. We consider a system $\Sigma$ which is described by the parabolic equation

$$
\dot{w}(t)+A w(t)=B v(t)+f(t), \quad \text { for a. a. } \quad t \in T, \quad w\left(t_{0}\right)=w_{0} .
$$

Here $A: V \rightarrow V^{*}$ is a linear continuous $\left(A \in \mathcal{L}\left(V ; V^{*}\right)\right)$ and symmetrical operator satisfying (for some $c_{*}>0$ and real $\omega$ ) the coercitivity condition

$$
\langle A w, w\rangle+\omega|w|_{H}^{2} \geq c_{*}|w|_{V}^{2} \quad \forall y \in V,
$$

$U$ is a Hilbert space, $f \in L_{2}(T ; H)$ is a given function, $|\cdot|_{H},|\cdot|_{U}$ and $|\cdot|_{V}$ stand for the norms in $H, U$ and $V$, respectively, $B: U \rightarrow H$ is a linear continuous operator $(B \in \mathcal{L}(U ; H))$. Let the following condition be fulfilled.

Condition 1. Operator $B$ is invertible.

Let $w\left(t_{0}\right)=w_{0} \in D\left(A_{H}\right)$, where $D\left(A_{H}\right)=\left\{w \in V: A_{H} \in H\right\}$. It is known that under such conditions, for any $v(\cdot) \in L_{2}(T ; U)$, there exists a unique solution $w(\cdot)=w\left(\cdot ; t_{0}, w_{0}, v(\cdot)\right)$ of equation (1) with the following properties [1]: $w(\cdot) \in W(T)=W^{1,2}(T ; H) \cap L_{2}(T ; V)$. Here, $W^{1,2}(T ; H)=\left\{w(\cdot) \in L_{2}(T ; H)\right.$ : $\left.\dot{w}(\cdot) \in L_{2}(T ; H)\right\}$, the derivative $\dot{w}(\cdot)$ is understood in the sense of distributions.

Assume that along with equation (10) we have another equation of the same form:

$$
\dot{x}(t)+A x(t)=B u(t)+f(t) \quad \text { for a.a. } \quad t \in T
$$

with an initial state $x\left(t_{0}\right)=x_{0} \in D\left(A_{H}\right)$. This equation (in what follows, we call it reference) is subject to the action of some reference control $u(\cdot) \in L_{2}(T ; U)$. The reference control as well as the corresponding solution $x(\cdot)=x\left(\cdot ; t_{0}, x_{0}, u(\cdot)\right)$ of equation (3) are a priori unknown. At discrete, frequent enough, time moments $\tau_{i} \in \Delta=\left\{\tau_{i}\right\}_{i=0}^{m}\left(\tau_{0}=t_{0}, \tau_{m}=\vartheta, \tau_{i+1}=\tau_{i}+\delta\right)$, the states $w\left(\tau_{i}\right)=$ $w\left(\tau_{i} ; t_{0}, w_{0}, v(\cdot)\right)$ of equation (11) as well as the states $x\left(\tau_{i}\right)=x\left(\tau_{i} ; t_{0}, x_{0}, u(\cdot)\right)$ of reference equation (3) are measured. The states $w\left(\tau_{i}\right)$ are measured with an error. The results of measurements are elements $\xi_{i}^{h} \in H$ satisfying the inequalities

$$
\left|w\left(\tau_{i}\right)-\xi_{i}^{h}\right|_{H} \leq h, \quad i \in[1: m-1] .
$$

Here, the value $h \in(0,1)$ is the measurement accuracy. It is required to design an algorithm for forming the control $v=v^{h}(\cdot)$ in equation (1) allowing us to track the solution $x(\cdot)$ of equation (3) by the solution $w(\cdot)$ of equation (11). Thus, we consider the problem consisting in constructing an algorithm, which (on the basis of current measurements of the values $w\left(\tau_{i}\right)$ and $x\left(\tau_{i}\right)$ ) forms in real time mode (by the feedback principle) the control $v=v^{h}(\cdot)$ in the righthand part of inequality (11) such that the deviation of $w(\cdot)=w\left(\cdot ; t_{0}, w_{0}, v^{h}(\cdot)\right)$ from $x(\cdot)=x\left(\cdot ; t_{0}, x_{0}, u(\cdot)\right)$ in metric of the space $C(T ; H) \cap L_{2}(T ; V)$ is small if the measurement accuracy $h$ is small enough. We also want the constructed algorithm to be resource-saving. This means that the resources of the synthetic 
control $v=v^{h}(\cdot)$ (i.e., the value $\left.\int_{t_{0}}^{\vartheta}\left|v^{h}(\tau)\right|_{U}^{2} d \tau\right)$ should exceed the resources of the reference control by a small value depending on the measurement accuracy $h$. This value tends to zero as $h$ tends to zero. Thus, we require the validity of the inequality

$$
\int_{t_{0}}^{\vartheta}\left|v^{h}(\tau)\right|_{U}^{2} d \tau \leq \int_{t_{0}}^{\vartheta}|u(\tau)|_{U}^{2} d \tau+\varphi(h)
$$

where $\varphi(h) \rightarrow 0$ as $h \rightarrow 0$.

In the case when the reference control $u$ as well as the control $v$ in inequality (11) are subject to instantaneous constraints $(u \in P, v \in P$, where $P \subset U$ is a given bounded and closed set), the problem above can be solved by means of the method of extremal shift [2]. Namely, if the control $v=v^{h}(\cdot)$ in the right-hand part of (1) is calculated by the formula

$$
v^{h}(t)=v\left(\tau_{i}, \xi_{i}^{h}, x\left(\tau_{i}\right)\right)=\arg \min \left\{\left(x\left(\tau_{i}\right)-\xi_{i}^{h}, B v\right): v \in P\right\} \text { for } t \in\left[\tau_{i}, \tau_{i+1}\right),
$$

then, as it follows from [3], for any $\varepsilon>0$ one can find numbers $h_{1}>0$ and $\delta_{1}>0$ such that the inequality

$$
\sup _{t \in T}\left|w\left(t ; t_{0}, w_{0}, v^{h}(\cdot)\right)-x\left(t ; t_{0}, x_{0}, u(\cdot)\right)\right|_{H} \leq \varepsilon
$$

is fulfilled if $h \in\left(0, h_{1}\right)$ and $\delta \in\left(0, \delta_{1}\right)$. The last inequality is valid for any reference control, i.e., for any Lebesgue measurable function $u(t) \in P$ for almost all $t \in T$. Here and below, we assume that $\omega>0$ and $w_{0} \in D \subset V$, where $D$ is a bounded set,

$$
\left|w_{0}-x_{0}\right|_{H} \leq h
$$

Thus, the method of extremal shift allows us to solve the problem of tracking the solution of the reference equation under instantaneous constraints on the controls $(v, u \in P)$. In the present paper, we assume that any function from the space $L_{2}(T ; U)$ can be the admissible control (both reference, $u(\cdot)$, and real, $v(\cdot))$. No additional information on the functions $v(\cdot)$ and $u(\cdot)$ is required. We construct a corresponding modification of the method of extremal shift, using, according to [4 9], the idea of its local regularization. Along with measuring the phase states at discrete time moments (see (4)), we also consider the case of "continuous" measuring of the states $x(t)$ and $w(t)$. Namely, it is assumed that, at every time $t \in T$, the phase states of equations (11) and (3) are measured; as a result, we have functions $\xi^{h}(t) \in H$ with the properties

$$
\left|\xi^{h}(t)-w(t)\right|_{H} \leq h, \quad t \in T .
$$

The functions $\xi^{h}(t), t \in T$, are Lebesgue measurable.

In control theory for distributed systems, a linear quadratic control problem (LQP) is widely known. Its solution methods have been studied rather well (see, for example, 11, 12 ). This problem consists in the minimization of some 
quadratic functional depending on a phase trajectory and control (for example, in the minimization of the deviations in $L_{2}$-norm from a reference control and state trajectory). The problem in question, which is in essence close to LQP, has, at the same time, several distinctive features. Among them, at the first turn, it is worth while noticing the following two features. Firstly, an LQP solution, as a rule, does not guarantee that we find a control generating a trajectory that is close to the reference trajectory in uniform metric. In addition, some apriori information on the reference control is rather often required. The second distinction is in the essence of solving methods. Namely, the method suggested in the present paper is based on constructions of the well-known in the theory of guaranteed control principle of extremal shift.

\section{Control Algorithm. Case of Continuous Measuring of Solutions}

First, we consider the case of "continuous" measuring of solutions of equations (11) and (3). In this case, inequalities (8) are valid (for simplicity, we set $\xi^{h}\left(t_{0}\right)=$ $\left.w_{0}\right)$. The problem consists in designing a rule forming (by the feedback principle) the control $v=v\left(t, \xi^{h}(t), w(t)\right)$. Fix a function $\alpha=\alpha(h):(0,1) \rightarrow(0,1)$. Let the control $v^{\alpha, h}(t)$ in equation (1) be defined by the formula

$$
v=\tilde{v}^{\alpha, h}(t)=v^{\alpha, h}(t)+\tilde{v}^{h}(t),
$$

where

$$
\tilde{v}^{h}(t)=c B^{-1}\left(x(t)-\xi^{h}(t)\right), \quad v^{\alpha, h}(t)=\alpha^{-1} B^{*}\left(x(t)-\xi^{h}(t)\right)
$$

Here, $B^{*}$ denotes the adjoint operator, $c=$ const $>2 \omega$. Thus, we obtain system (11), (3); i.e., we have the pair of equations

$$
\begin{gathered}
\dot{x}(t)+A x(t)=B u(t)+f(t), \\
\dot{w}^{\alpha, h}(t)+A w^{\alpha, h}(t)=\alpha^{-1} B B^{*}\left(x(t)-\xi^{h}(t)\right)+c\left(x(t)-\xi^{h}(t)\right)+f(t)
\end{gathered}
$$

with the initial condition $x\left(t_{0}\right)=x_{0}, \quad w^{\alpha, h}\left(t_{0}\right)=w_{0}$. Here, we denote by $w^{\alpha, h}(\cdot)$ the solution of equation (11) corresponding to the function $v=v^{\alpha, h}(\cdot)$ of form (9).

The second formula in (10) is an analog of relation (6). If the constraint in the form of the set $P$ is absent then the application of formula (6) for calculating the control $v$ is impossible, since in this case it is required to solve the problem of minimization of the linear functional $l_{i}(u)=\left(\xi_{i}^{h}-y\left(\tau_{i}\right), B u\right)$ over the whole space $U$. It is natural to replace this problem by a new regularized problem with a smoothing functional of the form $\alpha(h)|v|_{U}^{2}$, i.e., to replace problem (6) by the problem of finding the function $v^{\alpha, h}(t)$ by the rule

$$
v^{\alpha, h}(t)=\arg \min \left\{\alpha|v|_{U}^{2}-2\left(B^{*}\left(x(t)-\xi^{h}(t)\right), v\right)_{U}: v \in U\right\} .
$$


Formula (10) provides the solution of the new problem. Thus, to calculate $v^{\alpha, h}(t)$, we realize the regularization of the method of extremal shift by means of the method of smoothing functional, which is known in the theory of ill-posed problems.

Theorem 1. Let $\alpha=\alpha(h) \rightarrow 0$. Then the following inequalities

$$
\begin{gathered}
\left|x(t)-w^{\alpha, h}(t)\right|_{H}^{2}+2 c \int_{t_{0}}^{t}\left|x(\tau)-w^{\alpha, h}(\tau)\right|_{V}^{2} d \tau \leq d_{0}(h+\alpha(h)), \quad t \in T, \\
\int_{t_{0}}^{\vartheta}\left|\tilde{v}^{\alpha, h}(\tau)\right|_{U}^{2} d \tau \leq \int_{t_{0}}^{\vartheta}|u(\tau)|_{U}^{2} d \tau+d_{*}\left(h \alpha^{-2}(h)+h^{1 / 2}+\alpha^{1 / 2}(h)\right)
\end{gathered}
$$

are fulfilled. Here, $d_{0}, d_{*}=$ const $>0$ are constants, which do not depend on $h \in(0,1)$.

Proof. Due to (10), it holds that $\left|v^{\alpha, h}(t)\right|_{U}^{2} \leq 2 b^{2} \alpha^{-2}\left(h^{2}+\left|\mu_{\alpha, h}(t)\right|_{H}^{2}\right), t \in T$, where $\mu_{\alpha, h}(t)=x(t)-w^{\alpha, h}(t), b=\left|B^{*}\right|_{L(H ; U)}$ is the norm of the linear operator $B^{*} \in L(H ; U)$. In this case, we have

$$
\int_{t_{0}}^{t}\left|v^{\alpha, h}(\tau)\right|_{U}^{2} d \tau \leq 2 b^{2} \alpha^{-2} \varrho_{h}(t)+c_{1} h^{2} \alpha^{-2}, \quad \varrho_{h}(t)=\int_{t_{0}}^{t}\left|\mu_{\alpha, h}(\tau)\right|_{H}^{2} d \tau .
$$

Due to coercivity condition (2), we obtain,

$$
\begin{aligned}
\dot{\varepsilon}_{h}(t) & \leq-2\left(v^{\alpha, h}(t), B^{*}\left(x(t)-\xi^{h}(t)\right)\right)_{U}+\alpha\left|v^{\alpha, h}(t)\right|_{U}^{2}+2\left(u(t), B^{*}\left(x(t)-\xi^{h}(t)\right)\right)_{U}- \\
& -\alpha|u(t)|_{U}^{2}+2 b h\left\{|u(t)|_{U}+\left|v^{\alpha, h}(t)\right|_{U}\right\}+(2 \omega-c)\left|\mu_{\alpha, h}(t)\right|_{H}^{2}+2 c h^{2},
\end{aligned}
$$

where $\varepsilon_{h}(t)=\left|\mu_{\alpha, h}(t)\right|_{H}^{2}+2 c_{*} \int_{t_{0}}^{t}\left|\mu_{\alpha, h}(\tau)\right|_{V}^{2} d \tau+\alpha \int_{t_{0}}^{t}\left\{\left|v^{\alpha, h}(\tau)\right|_{U}^{2}-|u(\tau)|_{U}^{2}\right\} d \tau$. From (14) and (10), it follows that

$$
\varepsilon_{h}(t) \leq \varepsilon_{h}\left(t_{0}\right)+c_{2}+\int_{t_{0}}^{t} 2 b h\left\{|u(\tau)|_{U}+\left|v^{\alpha, h}(\tau)\right|_{U}\right\} d \tau+(2 \omega-c) \varrho_{h}(t) .
$$

From (13), (15), and the inequality $2 \omega-c<0$, we derive

$$
\varepsilon_{h}(t) \leq c_{3}\left(h+h^{3} \alpha^{-2}\right)+c_{4}\left(h \alpha^{-2}+h\right) \varrho_{h}(t) .
$$

Therefore, from (16) we get the bound

$$
\left|\mu_{\alpha, h}(t)\right|_{H}^{2} \leq c_{5}\left(h+\alpha+h^{3} \alpha^{-2}\right)+c_{4}\left(h \alpha^{-2}+h\right) \varrho_{h}(t) .
$$

By the Gronwall inequality and (17), we obtain

$$
\left|\mu_{\alpha, h}(t)\right|_{H}^{2} \leq c_{5}\left(h+\alpha+h^{3} \alpha^{-2}\right) \exp \left\{c_{4}\left(t-t_{0}\right)\left(h \alpha^{-2}+h\right)\right\} .
$$


Note that $h \alpha^{-2} \leq$ const. Then

$$
\left|\mu_{\alpha, h}(t)\right|_{H}^{2} \leq c_{6}(h+\alpha)
$$

From (16) and (19) we derive

$$
\varepsilon_{h}(t) \leq c_{3}\left(h+h^{3} \alpha^{-2}\right)+c_{7}\left(h \alpha^{-2}+1\right)(h+\alpha) \leq c_{9}(h+\alpha) .
$$

Relation (11) follows from (10). Let us verify (12). By virtue of inequality (19), from (16) we obtain

$$
\varepsilon_{h}(t) \leq c_{8}\left\{h+h^{3} \alpha^{-2}+\left(h \alpha^{-2}+h\right)(h+\alpha)\right\} \leq c_{9}\left\{h+h^{2} \alpha^{-2}+h \alpha^{-1}\right\} .
$$

Using (21), we get for $t \in T$

$$
\begin{gathered}
\int_{t_{0}}^{t}\left|v^{\alpha, h}(\tau)\right|_{U}^{2} d \tau \leq \int_{t_{0}}^{t}|u(\tau)|_{U}^{2} d \tau+c_{10} h \alpha^{-2}, \\
\left|\tilde{v}^{h}(t)\right|_{U} \leq c_{11}(h+\alpha)^{1 / 2} .
\end{gathered}
$$

Relation (12) follows from (22) and (23). The theorem is proved.

\section{Control Algorithm. Case of Discrete Measuring of Solutions}

Let us describe the algorithm for solving the problem in the case of discrete measuring of phase states. In this case, we assume that relations (4) are fulfilled. Let $l(\cdot): W^{1,2}(T ; H) \cap L_{2}(T ; V) \rightarrow \mathbb{R}^{+}, l(y(\cdot))=|y(\cdot)|_{C(T ; H)}+|\dot{y}(\cdot)|_{L_{2}(T ; H)}+$ $|y(\cdot)|_{L_{2}(T ; V)}$. In a standard way, we establish the validity of the following lemma.

Lemma 1. There exists a number $K=K\left(\omega, D, c_{*},|B|_{L(U ; H)}\right)$ such that the inequality $l\left(x\left(\cdot ; t_{0}, x, u(\cdot)\right)\right) \leq K\left(1+|u(\cdot)|_{L_{2}(T ; U)}\right)$ is fulfilled uniformly for any $x \in D$ and $u(\cdot) \in L_{2}(T ; U)$.

Let a family of partitions $\Delta_{h}=\left\{\tau_{h, i}\right\}_{i=0}^{m_{h}}, \tau_{h, 0}=t_{0}, \tau_{h, m_{h}}=\vartheta, \tau_{h, i+1}=\tau_{h, i}+$ $\delta(h)$ and a function $\alpha(h):(0,1) \rightarrow(0,1)$ be fixed. First, before the moment $t_{0}$, the value $h$ and the partition $\Delta_{h}$ of the interval $T$ are chosen and fixed. The work of the algorithm is decomposed into $m-1\left(m=m_{h}\right)$ identical steps. At the $i$ th step, which is carried out on the time interval $\delta_{i}=\left[\tau_{i}, \tau_{i+1}\right), \tau_{i}=\tau_{h, i}$, the following sequence of actions is fulfilled. First, at the moment $\tau_{i}$, the element

$$
v_{i}^{h}=\alpha^{-1} B^{*}\left(x\left(\tau_{i}\right)-\xi_{i}^{h}\right)
$$

is calculated. Then, the control defined by the formula

$$
v=\tilde{v}^{h}(t)=v_{i}^{h}+c B^{-1}\left(x\left(\tau_{i}\right)-\xi_{i}^{h}\right), \quad t \in \delta_{i},
$$


is fed onto the input of equation (1), where $c=$ const $>2 \omega$. Under the action of this control, instead of the state $w^{h}\left(\tau_{i}\right)=w^{h}\left(\tau_{i} ; \tau_{i-1}, w^{h}\left(\tau_{i-1}\right), v_{i-1}^{h}\right)$, the state $w^{h}\left(\tau_{i+1}\right)=w^{h}\left(\tau_{i+1} ; \tau_{i}, w^{h}\left(\tau_{i}\right), v_{i}^{h}\right)$ is realized. The work of the algorithm stops at the time moment $\vartheta$.

Let the family of partitions $\Delta_{h}$ of the time interval $T$ and the function $\alpha(h)$ have the following properties:

$$
\begin{gathered}
h \delta^{-1}(h) \leq C_{1}, \quad \delta(h) \alpha^{-2}(h) \rightarrow 0, \quad h \alpha^{-1}(h) \rightarrow 0, \\
\alpha(h) \rightarrow 0, \delta(h) \rightarrow 0 \text { as } h \rightarrow 0+.
\end{gathered}
$$

Here $C_{1}=$ const $>0$ is a constant, which does not depend on $h$.

Theorem 2. Uniformly with respect to $h \in(0,1)$, the inequalities

$$
\begin{gathered}
\lambda_{h}(t) \equiv\left|x(t)-w^{h}(t)\right|_{H}^{2}+2 c \int_{t_{0}}^{t}\left|x(\tau)-w^{h}(\tau)\right|_{V}^{2} d \tau \leq d_{1}(h+\alpha+\delta) \quad \forall t \in T, \\
\int_{t_{0}}^{\vartheta}\left|\tilde{v}^{h}(\tau)\right|_{U}^{2} d \tau \leq \int_{t_{0}}^{\vartheta}|u(\tau)|_{U}^{2} d \tau+d_{2}\left(h \alpha^{-1}+\delta \alpha^{-2}\right)+d_{3}(h+\alpha+\delta)^{1 / 2}
\end{gathered}
$$

are true. Here, $d_{1}, d_{3}\left(d_{1}-d_{3}=\right.$ const $\left.>0\right)$ are constants, which do not depend on $h, \alpha=\alpha(h)$, and $\delta=\delta(h)$.

Proof. First, we verify inequality (27). Using the invertibility of the operator $B$ as well as coercitivity condition (2), we obtain for a.a. $t \in \delta_{i}$ the inequality

$0.5 \frac{d\left|\mu^{h}(t)\right|_{H}^{2}}{d t}+c_{*}\left|\mu^{h}(t)\right|_{V}^{2}-\omega\left|\mu^{h}(t)\right|_{H}^{2} \leq\left(B\left(u(t)-v^{h}(t)\right)-c\left(x\left(\tau_{i}\right)-\xi_{i}^{h}\right), \mu^{h}(t)\right)_{U}$

where $\mu^{h}(t)=x(t)-w^{h}(t)$ for $t \in T, v^{h}(t)=v_{i}^{h}$ for $t \in \delta_{i}$. From the inequality

$c\left(\xi_{i}^{h}-x\left(\tau_{i}\right), \mu^{h}(t)\right) \leq-0.5 c\left|\mu^{h}(t)\right|_{H}^{2}+4 c h^{2}+8 c\left(t-\tau_{i}\right) \int_{\tau_{i}}^{t}\left\{|\dot{x}(\tau)|_{H}^{2}+\left|\dot{w}^{h}(\tau)\right|_{H}^{2}\right\} d \tau$

we have for a.a. $t \in \delta_{i}$

$$
\begin{gathered}
\left(B\left(u(t)-v^{h}(t)\right)-c\left(x\left(\tau_{i}\right)-\xi_{i}^{h}\right), \mu^{h}(t)\right)_{U} \leq \\
\left(B\left(u(t)-v^{h}(t)\right), x\left(\tau_{i}\right)-\xi_{i}^{h}\right)_{U}+\varrho_{i}(t, h)+\chi_{i}(t, h)-0.5 c\left|\mu^{h}(t)\right|_{H}^{2} .
\end{gathered}
$$

Here,

$$
\chi_{i}(t, h)=4 c h^{2}+8 c\left(t-\tau_{i}\right) \int_{\tau_{i}}^{t}\left\{|\dot{x}(\tau)|_{H}^{2}+\left|\dot{w}^{h}(\tau)\right|_{H}^{2}\right\} d \tau
$$




$$
\varrho_{i}(t, h)=b\left(|u(t)|_{U}+\left|v^{h}(t)\right|_{U}\right)\left(h+\int_{\tau_{i}}^{t}\left\{\left|\dot{w}^{h}(\tau)\right|_{H}+|\dot{x}(\tau)|_{H}\right\} d \tau\right) .
$$

For a.a. $t \in \delta_{i}$, we deduce that

$$
\begin{gathered}
\dot{\varepsilon}^{h}(t) \leq-2\left(v^{h}(t), B^{*}\left(x\left(\tau_{i}\right)-\xi_{i}^{h}\right)\right)_{U}+\alpha\left|v^{h}(t)\right|_{U}^{2}+ \\
+2\left(u(t), B^{*}\left(x\left(\tau_{i}\right)-\xi_{i}^{h}\right)\right)_{U}-\alpha|u(t)|_{U}^{2}+2 \varrho_{i}(t, h)+2 \chi_{i}(t, h)+(2 \omega-c)\left|\mu^{h}(t)\right|_{H}^{2},
\end{gathered}
$$

where $\varepsilon^{h}(t)=\left|\mu^{h}(t)\right|_{H}^{2}+2 c_{*} \int_{t_{0}}^{t}\left|\mu^{h}(\tau)\right|_{V}^{2} d \tau+\alpha \int_{t_{0}}^{t}\left\{\left|v^{h}(\tau)\right|_{U}^{2}-|u(\tau)|_{U}^{2}\right\} d \tau$. Therefore, by virtue of the rule of forming the control $\tilde{v}^{h}(\cdot)$ (see (24) and (25)), we conclude that, for a.a. $t \in \delta_{i}$,

$$
\begin{aligned}
& \varepsilon^{h}(t) \leq \varepsilon^{h}\left(\tau_{i}\right)+c_{1} h^{2}+c_{2} \delta \int_{\tau_{i}}^{t}\left\{|u(\tau)|_{U}^{2}+\left|v^{h}(\tau)\right|_{U}^{2}\right\} d \tau+ \\
& +c_{3} \delta \int_{\tau_{i}}^{t}\left\{\left|\dot{w}^{h}(\tau)\right|_{H}^{2}+|\dot{x}(\tau)|_{H}^{2}\right\} d \tau+(2 \omega-c) \int_{\tau_{i}}^{t}\left|\mu^{h}(\tau)\right|_{H}^{2} d \tau .
\end{aligned}
$$

Summing the right-hand and left-hand parts of (29) over $i$ and taking into account Lemma 1, we obtain for $t \in T$

$$
\varepsilon^{h}(t) \leq \varepsilon^{h}\left(t_{0}\right)+c_{4} h^{2} \delta^{-1}+c_{6} \delta+c_{7} \gamma_{h, \delta}(t) .
$$

Here, $\gamma_{h, \delta}(t)=\delta^{2} \sum_{j=0}^{i(t)}\left|v_{j}^{h}\right|_{U}^{2}$. Using (4) and the rule of forming $v_{i}^{h}$ (see (24)), we get

$$
\left|v_{i}^{h}\right|_{U}^{2} \leq 2 b^{2}\left(\varrho_{i}^{h}+h^{2}\right) \alpha^{-2} \leq c_{8}\left(\varrho_{i}^{h}+h^{2}\right) \alpha^{-2},
$$

where $\varrho_{i}^{h}=\left|x\left(\tau_{i}\right)-w^{h}\left(\tau_{i}\right)\right|_{H}^{2}$. Due to (7), we have $\varepsilon^{h}\left(t_{0}\right) \leq h^{2}$. Therefore, we derive from (30) the estimate

$$
\lambda_{h}(t) \leq c_{9}\left(\delta+h^{2} \delta^{-1}+\alpha+\gamma_{h, \delta}(t)\right) .
$$

Note that $\varrho_{i}^{h} \leq \lambda_{i}^{h}$, where $\lambda_{j}^{h}=\lambda_{h}\left(\tau_{j}\right)$. Therefore, for $t \in\left[\tau_{i}, \tau_{i+1}\right]$, due to (31), the inequality

$$
\gamma_{h, \delta}(t) \leq c_{8} \delta^{2} \sum_{j=0}^{i(t)}\left(\lambda_{j}^{h}+h^{2}\right) \alpha^{-2}
$$

is valid. Consequently, (32) implies the inequality

$$
\lambda_{i}^{h} \leq c_{10}\left(\delta+h^{2} \delta^{-1}+\alpha\right)+c_{11} \delta h^{2} \alpha^{-2}+c_{12} \delta^{2} \alpha^{-2} \sum_{j=0}^{i} \lambda_{j}^{h} .
$$

By the discrete Gronwall inequality [10, (34), and the inequalities $h \delta^{-1}(h) \leq C_{1}$, $\delta \alpha^{-2}(h) \leq C_{2}$ as $h \rightarrow 0$ (see (26) $)$, we have

$$
\lambda_{i}^{h} \leq c_{14}(h+\delta+\alpha), \quad i \in[0: m] .
$$


This and (33) imply $\gamma_{h, \delta}(t) \leq c_{15}(h+\delta+\alpha) t \in T$. Moreover, from the last inequality and (32), we have $\lambda_{h}(t) \leq c_{16}\left(\delta+h^{2} \delta^{-1}+\alpha+\gamma_{h, \delta}(t)\right) \leq c_{17}(h+\delta+\alpha)$. Relation (27) follows from the last inequality. The proof of (28) is similar to the proof of (12). The theorem is proved.

It follows from Theorems 1 and 2 that the algorithms presented above are resource-saving.

\section{Example}

The second algorithm was tested. The parabolic equation

$$
w_{t}(t, \eta)-\partial^{2} w(t, \eta) / \partial \eta^{2}=v(t, \eta), \quad \eta \in[0,1]
$$

with the boundary $w(t, 0)=w(t, 1), t \in T=[0,2]$ and initial $w(0, \eta)=0$, $\eta \in[0,1]$ conditions was considered. The reference equation (see (3) ) was of the form

$$
\begin{gathered}
x_{t}(t, \eta)-\partial^{2} x(t, \eta) / \partial \eta^{2}=u(t, \eta), \quad \eta \in[0,1] \\
x(t, 0)=x(t, 1)=0, \quad t \in T, \quad x(0, \eta)=0, \quad \eta \in[0,1] .
\end{gathered}
$$

Equations (35) and (36) were solved by the grid method 10. The grid $\left\{\eta_{j}\right\}_{j=0}^{n}$, $\eta_{0}=0, \eta_{n}=1$ with the step $\gamma_{N}=1 / n$ was taken on the interval $[0,1]$. The control $v=v^{h}(t, \eta)$ in the right-hand part of (35) was calculated by formula (25) taking the form

$$
v^{h}\left(t, \eta_{j}\right)=\left(\alpha^{-1}+c\right)\left(x\left(\tau_{i}, \eta_{j}\right)-\xi_{i}^{h}\left(\eta_{j}\right)\right), \quad t \in\left[\tau_{i}, \tau_{i+1}\right), \quad j \in[0: n] .
$$

During the experiment, we assumed that $\xi_{i}^{h}\left(\eta_{j}\right)=w\left(\tau_{i}, \eta_{j}\right)+h$. In figs. 1-4, the cross-sections of the trajectories $x$ (dashed line) and $w$ (solid line) by the hyperplane $\eta=0,4$ are presented, as well as the variations of the values $p(t)=$ $\int_{0}^{t}\left|v^{h}(\tau)\right|_{L_{2}([0,1])}^{2} d \tau$ (solid line) and $q(t)=\int_{0}^{t}\left|u^{h}(\tau)\right|_{L_{2}([0,1])}^{2} d \tau$ (dashed line). Figs. 1 and 3 correspond to the case $\delta=2 / m_{h}, m_{h}=800, n=10, h=0.05$;

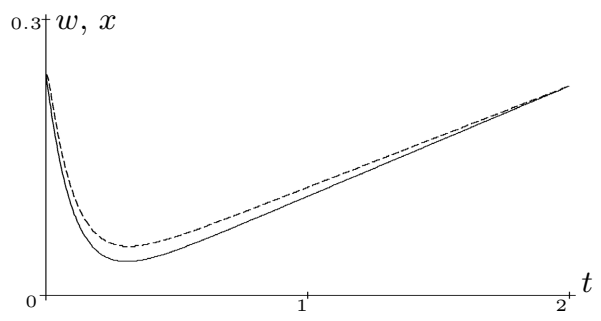

Fig. 1.

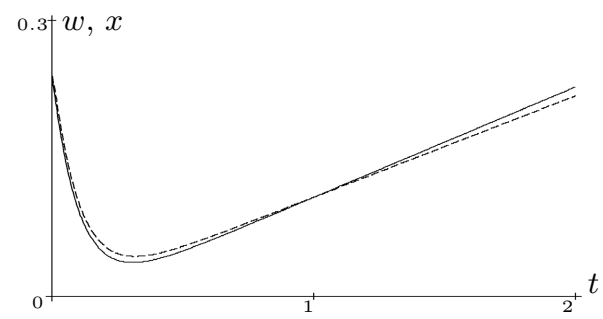

Fig. 2. 


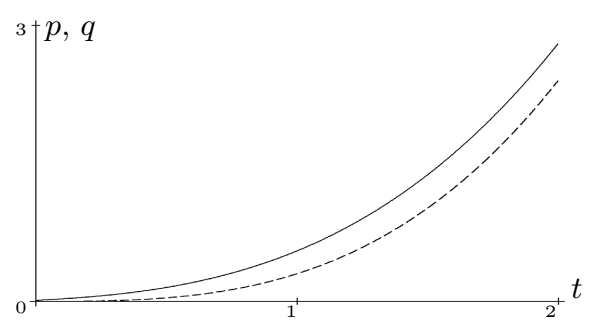

Fig. 3.

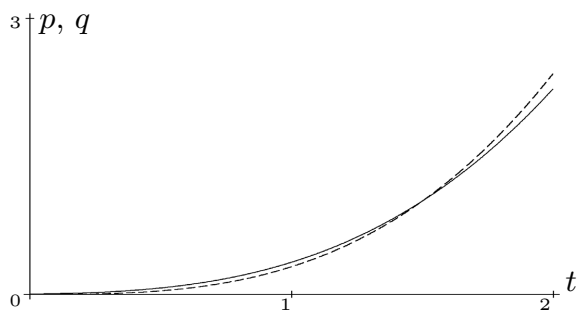

Fig. 4.

figs. 2 and 4 , to the case $\delta=2 / m_{h}, m_{h}=800, n=10, h=0.01$. As the numerical experiment showed,

$$
\max _{\substack{i \in\left[0: m_{h}\right] \\ j \in[0: n]}}\left|x\left(\tau_{i}, \eta_{j}\right)-w^{h}\left(\tau_{i}, \eta_{j}\right)\right|= \begin{cases}0,01926, & \text { in the first case } \\ 0,00972, & \text { in the second case. }\end{cases}
$$

\section{References}

1. Bensoussan, A., Da Prato, G., Delfour, M., Mitter, S.: Representation and Control of Infinite Dimensional Systems. Birkhauser, Boston (1992)

2. Krasovskii, N.N., Subbotin, A.I.: Game-theoretical Control Problems. Springer, New-York (1988)

3. Vaysburd, I.F., Osipov, Y.S.: Differential pursuit game for systems with distributed parameters. Prikl. Mat. Mech. 39(5), 772-779 (1975) (in Russian)

4. Osipov, Y.S., Kryazhimskii, A.V.: Inverse Problems for Ordinary Differential Equations: Dynamical Solutions. Gordon and Breach, London (1995)

5. Maksimov, V.I.: Dynamical Inverse Problems of Distributed Systems. VSP, Utrecht (2002)

6. Osipov, Y.S., Kryazhimskii, A.V., Maksimov, V.I.: Methods of Dinamical Reconstruction of Inputs of Controlled Systems. Ekaterinburg (2011) (in Russian)

7. Maksimov, V., Pandolfi, L.: Dynamical reconstruction of inputs for constraction semigroup systems: the boudary input case. J. Optim. Theor. Appl. 103, 401-420 (1999)

8. Maksimov, V., Pandolfi, L.: On reconstruction of unbounded controls in nonlinear dynamical systems. Prikl. Mat. Mech. 65(3), 35-42 (2001) (in Russian)

9. Maksimov, V., Tröltzsch, F.: Dynamical state and control reconstruction for a phase field model. Dynamics of Continuous, Discrete and Impulsive Systems. A: Mathematical Analysis 13(3-4), 419-444 (2006)

10. Samarskii, A.A.: Introduction to the Theory of Difference Schemes. Nauka, Moscow (1971) (in Russian)

11. Lasiecka, I., Triggiani, R.: Control theory for partial differential equations: continuous and approximation theories. I. Abstract parabolic systems, Cambridge (2000)

12. Curtain, R.F., Pritchard, A.J.: Infinite dimensional linear systems theory. LNCIS, vol. 8. Springer (1978) 